\title{
Ultrasound for the Diagnosis of primary thyroid lymphoma
}

\author{
Pan Zhang ${ }^{1}$, Ying Wang ${ }^{1}$, Yu Dong ${ }^{1}$, Zhuo Zhang ${ }^{2 *}$ and Guang-Sen $\mathrm{Li}^{{ }^{1 *}}$ \\ ${ }^{1}$ Department of Ultrasound, the Second Affiliated Hospital of Dalian Medical University, Dalian, China \\ ${ }^{2}$ Department of Radiation Oncology, the Second Affiliated Hospital of Dalian Medical University, Dalian, China
}

\begin{abstract}
Objective: The purpose of this work is to draw attention to the characteristic sonographic features of Primary Thyroid Lymphoma (PTL) through analysing 15 cases.

Methods: Retrospectively analysed the ultrasonographic features of 15 PTL cases ( 2 males, 13 females), who were confirmed by intraoperative pathologic or ultrasound-guided biopsy, and in the context of clinical manifestations and ultrasound picture support its diagnosis.

Results: All 15 patients were diagnosed as diffuse large B-cell lymphoma of non-Hodgkin's lymphoma by histopathological analysis. There were three diffuse PTL $(3 / 15,20 \%)$, which were characterized with a dramatically increased of the thyroid gland volume, diffuse structural changes, marked hypoechogenicity, heterogeneous echotexture, unclear boundaries and one case with posterior acoustic enhancement, no microcalcification, and one cervical lymphadenopathy. There were 10 cases of unilateral thyroid node and 2 cases with bilateral node in the remainder 12 nodular PTL cases (12/15, 80\%). There were 7 heterogeneous echotextures and 3 homogeneous echotexture in the 10 cases with unilateral goiter. And the lesions of the 2 bilateral goiters were with homogeneous echotexture. All lesions were with irregular shapes, clear boundaries, and hypoechogenicity. There were 3 cases with microcalcification, 8 cases with hyperechoic posterior shadowing, and 2 cases with cervical lymphadenopathy. A Colour Doppler was utilized to evaluate intralesional blood flow. Eleven cases showed abundant flow in the lesion, mainly were high frequency and high resistance artery blood flow, and 4 cases showed no abnormal blood flow.
\end{abstract}

Conclusions: although the characteristics are not very typical, most of the lesions are rich in blood flow, mainly high frequency and high resistance artery blood flow, and PTL can be diagnosed initially by ultrasonography combined with clinical manifestation.

\section{Introduction}

Primary Thyroid Lymphoma (PTL) is defined as lymphoma involving either the thyroid gland alone or the thyroid gland and adjacent neck lymph nodes without contiguous spread or distant metastases at the time of diagnosis [1]. PTL is a rare disease accounting for $0.6 \%-5.0 \%$ of all thyroid malignancies, and accounting approximately $3 \%$ of extranodal lymphomas [2]. Additionally, PTL is more common in older females [3]. Prompt therapies, such as chemotherapy, radiotherapy, or surgery should be pursued as soon as possible after diagnosis. However, it is difficult to differentiate PTL from other thyroid diseases by ultrasonography alone, because the ultrasonographic features of PTL are variable and complicated [4]. In our study, we retrospectively reviewed 15 cases of PTL evaluated with ultrasonography to provide support for the early diagnosis of PTL.

\section{Material and methods}

\section{Study population}

In this retrospective series approved by our Institutional Review Board, between January 2005 and August 2016, fifteen patients with PTL were diagnosed in our hospital. The ratio of male to female patients was 2:13, their ages ranged from 52 to 84 years with a median age of 69 years. All 15 patients had non-Hodgkin's lymphoma of B cell origin, Diffuse Large B-Cell Lymphoma (DLBCL). Eight patients had stage IE, six patients had stage IIE, and one patients had stage IIIE. After diagnosis, 8 cases received surgery and late chemotherapy; 6 cases received systemic chemotherapy combined with local radiotherapy, mainly CHOP-R or COP-R protocols; and 1 patient received chemotherapy alone (a-COP protocol). In this study, all patients underwent ultrasound examination. All 15 cases presented with an anterior neck mass. Additionally, clinical symptoms included 1 patient with pain and night sweats, 1 patient with dysdipsia, 5 patients with hoarseness of voice, 2 patients with dyspnea and 1 patient with dysphagia. A total of 7 cases had symptoms of compression, 3 cases with cervical lymphadenectasis, 2 cases with chronic lymphocytic thyroiditis and hypothyroidism.

\section{Methods}

All sonograms were obtained with 5-12-MHz linear transducers (Philips Healthcare, Amsterdam, Holland). All patients were scanned in a supine position with the neck hyperextended, fully exposing the anterior cervical region, imagesn from the sonographic studies were evaluated for the size of the thyroid gland, the echoes of the thyroid gland, and the mass. If a mass was observed, the size, shape, boundaries, internal echoes, posterior echoes, and the presence of calcifications were noted. The cervical lymph nodes, and other surrounding structures, including the tracheal and larynx were evaluated. The

Correspondence to: Guang-Sen Li, Department of Ultrasound, the Second Affiliated Hospital of Dalian Medical University, Dalian, China, Postal code: 116027, Tel: +8617709870319, E-mail: liguangsen009@163.com

Zhuo Zhang, Department of Radiation Oncology, the Second Affiliated Hospital of Dalian Medical University, Dalian, China, Postal code: 116027, Tel: +8617709873899; E-mail: 799832582@qq.com

Key words: Non-Hodgkin's lymphoma, primary thyroid lymphoma, sonography

Received: January 18, 2018; Accepted: February 08, 2018; Published: February 12,2018 
diagnosis of primary thyroid lymphoma was made histopathologically in all cases using tissue obtained during surgery in 8 cases ( 4 total thyroidectomy, 4 lobectomy), and via ultrasound-guided biopsy in 7 cases ( 5 fine-needle aspiration, 2 core biopsy). The diagnosis of lymphadenectasis was made histopathologically in 3 cases using tissue obtained by ultrasound-guided biopsy. Ultrasonographic features were retrospectively determined by two ultrasonographers, both of whom had over 15 years of experience in thyroid imaging.

\section{Results}

\section{Pathological results}

Histopathological samples were analysed with immunohistochemistry, which provided the diagnosis of nonHodgkin's lymphoma of B cell origin, diffuse large B-cell lymphoma (DLBCL). Immunohistochemistry analysis showed that antigens (CD20, CD22, and CD79a) [5] associated with B cells were positive in the tissues, suggesting B-cell lymphoma (Figures 1 and 2). There were 3 cases of cervical lymph node enlargement identified by ultrasonography that also proved to be cervical lymph node metastases.

\section{Ultrasonic characteristics}

In our study, there were three diffuse PTL $(3 / 15,20 \%)$, which were characterized with a dramatically increased of the thyroid gland volume, presence of normal thyroid echoes, diffuse structural changes, marked hypoechogenicity, heterogeneous echotexture, unclear boundaries, and one case with posterior acoustic enhancement (Figure 3). None of these 3 patients had evidence of microcalcification, and one of these patients had cervical lymphadenopathy. There were 10 cases of unilateral thyroid node and 2 cases with bilateral node in the left 12 nodular PTL cases $(12 / 15,80 \%)$, they all presented normal thyroid echoes. There were 7 heterogeneous echotextures and 3 homogeneous echotexture in the 10 cases with unilateral goiter. And the lesions of the two bilateral goiters were with homogeneous echotexture. The maximal lesion size was $68 \mathrm{~mm} \times 39 \mathrm{~mm} \times 61 \mathrm{~mm}$, and the smallest lesion was $28 \mathrm{~mm} \times 10 \mathrm{~mm} \times 4 \mathrm{~mm}$. All lesions were with irregular shapes, clear boundaries, and hypoechogenicity (Figure 4 ). There were 3 cases with microcalcification, 8 cases with hyperechoic posterior shadowing, and 2 cases with cervical lymphadenopathy. No patients had mixed PTL $(0 / 15,0 \%)$. A Colour Doppler was utilized to evaluate intralesional blood flow. Eleven cases showed abundant flow in the lesion, mainly

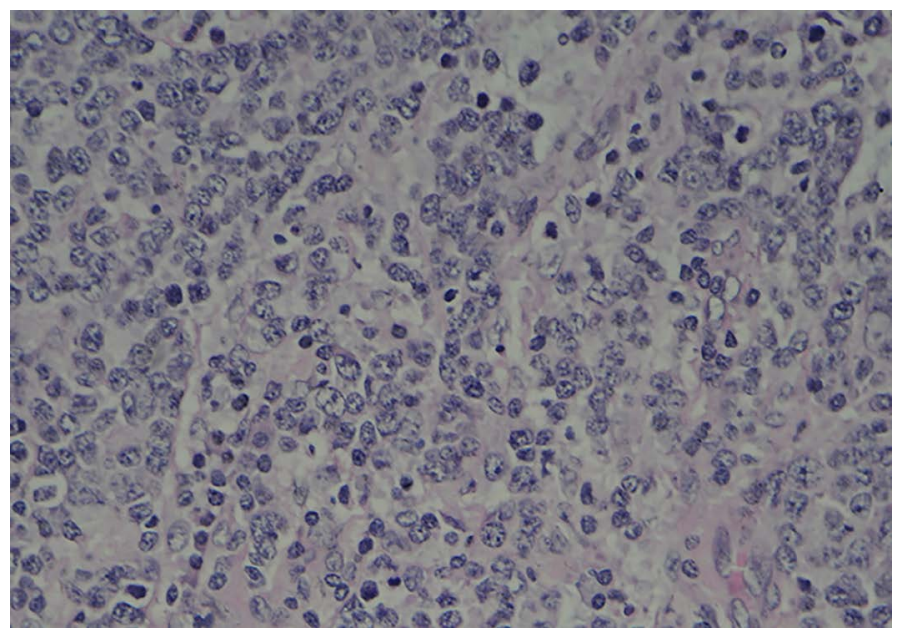

Figure 1. Pathological results. Pathological confirmation of non-Hodgkin's lymphoma by diffuse proliferation of large atypical lymphocytes, and large nuclei with coarse nuclear reticulum, (Eosin and hematoxylin stain, magnification, $\times 400)$

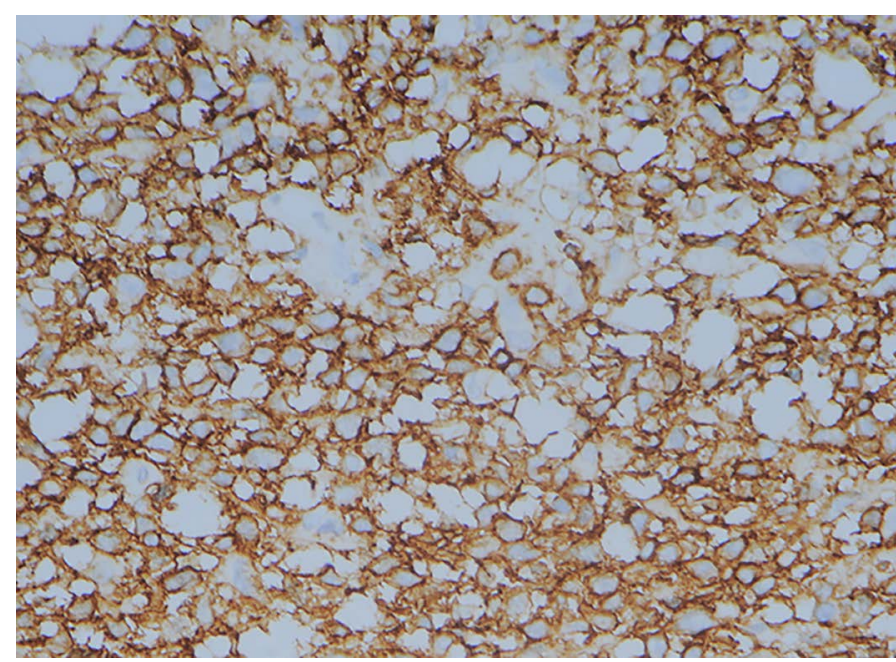

Figure 2. Pathological results. Immunohistochemical analysis revealed that antigens associated with B cells, such as cluster of differentiation 20 (CD 20), were widely expressed. (Magnification, $\times 400$ )

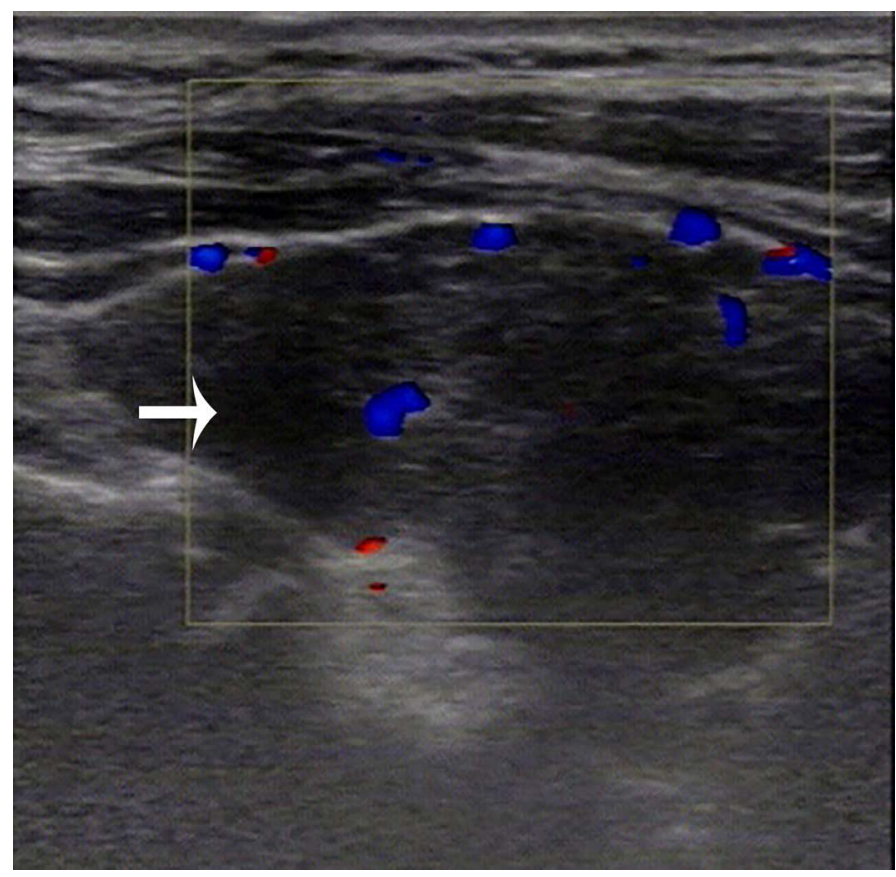

Figure 3. Diffuse PTL: the white arrow indicated the lesion, which was marked hypoechogenicity, heterogeneous echotexture, and unclear boundaries. Color Doppler image shows increased blood flow in the lesion

high frequency and high resistance artery blood flow, and 4 cases showed no abnormal blood flow.

The correct rate of ultrasound diagnosis: In our 15 cases, there were only 2 cases (13.3\%) diagnosed as PTL via ultrasound. Thirteen cases were misdiagnosed: three were misdiagnosed as Hashimoto's thyroiditis (20\%), six were misdiagnosed as cancerous goiter (40\%), and four were misdiagnosed as nodular goiter (26.7\%).

\section{Discussion}

PTL is a rare disease, accounting for $0.6 \%-5.0 \%$ of all thyroid malignancies, and approximately $3 \%$ of extranodal lymphoma [2]. It is 2 to 8 times more common in women than in men [6], and most PTL patients are diagnosed at the age of 60 to 70 years old [7]. PTL is divided 


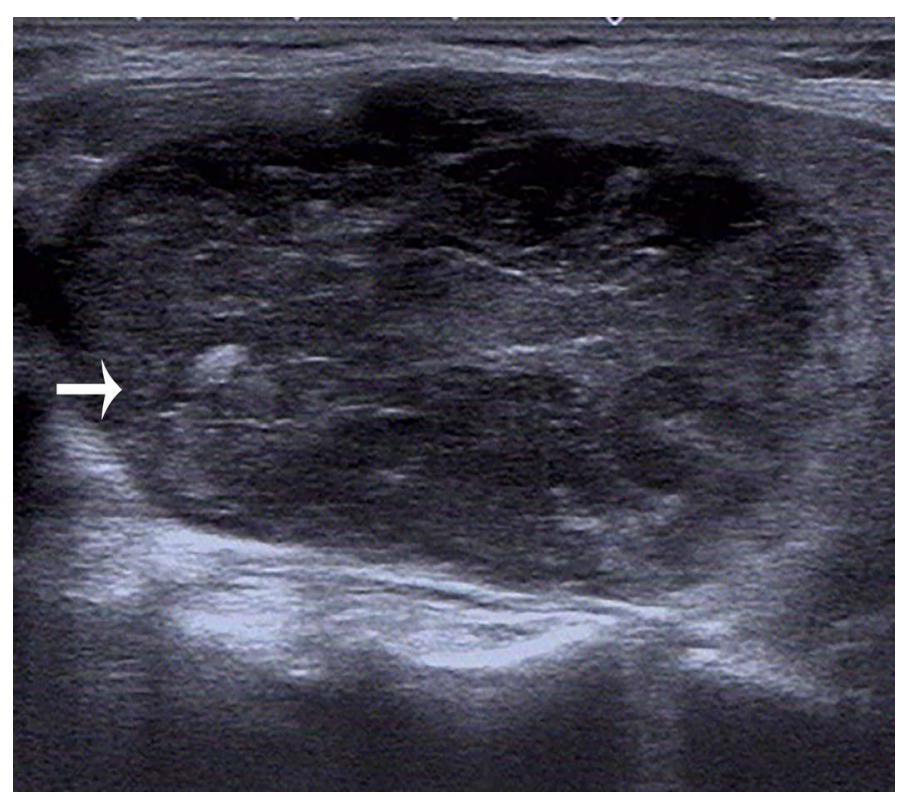

Figure 4. Nodular PTL: the white arrow indicated the lesion, which was irregular shapes, clear boundaries, and hypoechogenicity, exhibited calcification spots and posterior acoustic enhancement

into two common histological subtypes: non-Hodgkin's lymphoma of B cell origin, diffuse large B-cell lymphoma and Mucosa-Associated Lymphoid Tissue (MALT) lymphomas [8], MALT lymphomas account for $23 \%$ to $47 \%$ of PTL $[9,10]$. DLBCL tends to be more aggressive and has a worse prognosis than MALT lymphomas [11,12]. However, previous reports described MALT lymphomas occasionally progressed to highgrade lymphomas DLBCL $[13,14]$. All 15 patients in this case series were diagnosed as DLBCL. The pathogenesis of PTL is not clear, and it may be related to autoimmune disease or chronic inflammatory disease [15]. The risk of patients with Hashimoto's thyroiditis developing PTL is 40 to 80 times than that of the general population [1]. Watanabe et al. identified Hashimoto's thyroiditis in $80 \%$ of primary thyroid lymphomas, though only $0.6 \%$ of patients with Hashimoto's thyroiditis developed into PTL. Patients with lymphocytic thyroiditis should be regularly followed for the development of lymphoma [9]. The most common clinical manifestation of PTL is painless and rapidly growing of goiter, which is often the first presenting complaint, however, other compressive symptoms, including hoarseness of voice, dysphasia, stridor, dyspnea, and dysphagia can also occur [16]. Some patients may also develop systemic symptoms such as fever, weight loss, or night sweats, although these are uncommon. Diagnosis of PTL mainly depends on imaging features and histopathological examination. Obtaining a definitive diagnosis early is important, because PTL is curable if treated in timely manner [17]. The main treatments for PTL are surgery, radiotherapy, chemotherapy, or a combination of them. In recent years, combination locoregional radiation and chemotherapy has been recognized as an effective treatment for PTL $[18,19]$. Imaging findings associated with pathologic and immunohistochemical findings may guide therapeutic planning and predicting prognosis [20]. When the PTL is suspected showed by ultrasound, the primary examination is fine needle aspiration, while Fine-Needle Aspiration Cytology (FNAC) is appropriate for obtaining neoplastic cells from a markedly hypoechoic area in segmental or nodular PTL, and core needle biopsy or surgery are more valuable in diagnosing in the diffuse type, as it can provide enough tissue needed for the exact diagnosis of PTL $[21,22]$. Some scholars have found that FNAC combined with flow cytometry, has a sensitivity and specificity for detecting B cell lymphoma of $97 \%$ and $87 \%$, respectively [23]. However, due the amount of sample required for flow cytometry, its function is limited [24].

With the gradual increase of thyroid nodules in patients, ultrasound examination has become the first choice for the diagnosis of benign and malignant thyroid nodules and plays a significant role in both initial diagnosis and follow up. According to the ultrasonographic classification scheme proposed by Ota et al. [25], primary thyroid lymphoma can be divided into three types. The first is the diffuse type, with main characteristics of a bilaterally enlarged thyroid gland, generally low echoes, heterogeneous, and unclear boundaries between the lesion and normal thyroid gland. The second is the nodular type, with main characteristics of single or multiple nodules in the thyroid, irregular boundaries, and a homogeneous, hypoechoic appearance, equal echoic, or similar to a cyst-like structure. The third is the mixed type, which contains the characteristics of nodular and diffuse PTL. While in the study by Wang et al. [21] showed heterogeneous hypoechoic in nodular PTL. The cases in this study conformed to the above-mentioned ultrasonic characteristics, it was also observed that diffuse-type PTL and Hashimoto's thyroiditis were similar in ultrasonographic features. While the blood flow velocity of superior thyroid artery in PTL is within the normal range, and there is normal thyroid tissue in PTL. The nodular PTL is usually with posterior acoustic enhancement and normal thyroid tissue. Compared with nodular goiter, PTL is rich in central-blood flow, mainly high frequency and high resistance artery blood flow, this may be helpful in distinguishing the two diseases from ultrasonography [21]. Sonographic features of PTL were correlated with pathologic in studies, the hypoechoic areas of PTL are consistent with lymphoepithelial lesion containing neoplastic cells, with uniformly diffuse distributed large highly heterogeneous immature lymphoma cells [26,27]. In PTL, lymphoma cells grow diffusely toward reactive follicular center and follicular region, leading to diffuse PTL hypoechoic lesion with unclear boundaries. Pathologically, microcalcification is a psammoma body that is comprised of $10-100 \mu \mathrm{m}$ round, laminar, crystalline, calcific deposits [28]. Heterogeneous echoes are consistent with fibrosis at pathologic examination. There are dense colloid bodies around the lymphoma cells, and the surrounding thyroid tissue often has atrophy and fibrosis changes. If the fibrous bands in the lesion are relatively thin, the ultrasound image would be similar to the linear echogenic stands pattern; if the fibrous bands are dominant in the specimens, the ultrasound pattern would range from a dense linear echogenic strands pattern to a segmental pattern [26]. At different stages of development, there may be necrosis, hemorrhage and accumulation of colloid bodies in the lesions, which may cause heterogeneous echo pattern [29]. While homogeneous markedly hypoechoic mass rarely associated with necrosis [19]. Because of the small acoustic impedance and low echo spectrum of accumulated large amounts of immature lymphocytes in PTL and a small amount of fibrous bands in the lesion, the easy penetration of ultrasound in lymphoma causes the enchancement of posterior echoes. The sonographic findings of PTL are different from each type, which may be related to the different stages of the disease [30].

In our 15 cases, there were only 2 cases diagnosed as PTL via ultrasound, thirteen cases were misdiagnosed, the main reasons might be that PTL is so rarely that the disease is not clearly understood, its imaging features are not unique, and some patients may have concurrent Hashimoto's thyroiditis. Therefore, PTL can sometimes prove to be a diagnostic challenge. The main clinical manifestation of PTL is a rapidly enlarging thyroid mass, in the seventh decade, and is more common in 
women [30]. The diagnosis of PTL was made histopathologically in 7 cases using tissue obtained by ultrasound-guided biopsy, that provide the basis for the choice of clinical treatment, and to avoid unnecessary surgical resection of individual patients.

\section{Conclusion}

In conclusion, ultrasonographic features of PTL are very atypical, and ultrasonography has some limitations in the diagnosis of PTL, while ultrasonographic examination combined with the clinical manifestations is very important for the initial diagnosis of PTL, and ultrasound guided biopsy and pathological examination is required for definitive diagnosis of PTL.

\section{Compliance with ethical standards \\ Ethical approval}

All procedures performed in studies involving human participants were in accordance with the ethical standards of the institutional and national research committee and with the 1964 Helsinki declaration and its later amendments or comparable ethical standards. This article does not contain any studies with animals performed by any of the authors.

\section{Informed consent}

Informed consent was obtained from all individual participants included in the study.

\section{Funding}

This research did not receive any specific grant from funding agencies in the public, commercial, or not-for-profit sectors.

\section{References}

1. Ansell SM, Grant CS, Habermann TM (1999) Primary thyroid lymphoma. Semin Oncol 26: 316-323. [Crossref]

2. Walsh S, Lowery AJ, Evoy D, McDermott EW, Prichard RS (2013) Thyroid lymphoma: recent advances in diagnosis and optimal management strategies. Oncologist 18: 994 1003. [Crossref]

3. Zinzani PL, Magagnoli M, Galieni P, Martelli M, Poletti V, et al. (1999) Nongastrointestinal low-grade mucosa-associated lymphoid tissue lymphoma: analysis of 75 patients. J Clin Oncol 17: 1254. [Crossref]

4. Basu S, Li G, Bural G, Alavi A (2009) Fluorodeoxyglucose positron emission tomography (FDG-PET) and PET/computed tomography imaging characteristics of thyroid lymphoma and their potential clinical utility. Acta Radiol 50: 201-204. [Crossref]

5. Wang JH, Chen L, Ren K (2014) Identification of primary thyroid lymphoma with medical imaging: A case report and review of the literature. Oncol Lett 8: 2505-2508. [Crossref]

6. Dundar HZ, Sarkut P, Kirdak T, Korun N (2015) Primary thyroid lymphoma. Ulus Cerrahi Derg 32: 75-77. [Crossref]

7. Xia Y, Wang L, Jiang Y, Dai Q, Li X, et al. (2014) Sonographic appearance of primary thyroid lymphoma-preliminary experience. PLoS One 9: e114080. [Crossref]

8. Derringer GA, Thompson LD, Frommelt RA, Bijwaard KE, Heffess CS, et al. (2000) Malignant lymphoma of the thyroid gland: a clinicopathologic study of 108 cases. $\mathrm{Am}$ J Surg Pathol 24: 623-639. [Crossref]

9. Watanabe N, Noh JY, Narimatsu H, Takeuchi K, Yamaguchi T, et al. (2011) Clinicopathological features of 171 cases of primary thyroid lymphoma: a long-term study involving 24553 patients with Hashimoto's disease. Br J Haematol 153: 236-243. [Crossref]

10. Thieblemont C, Mayer A, Dumontet C, Barbier Y, Callet-Bauchu E, et al. (2002) Primary thyroid lymphoma is a heterogeneous disease. J Clin Endocrinol Metab 87: 105-111. [Crossref]
11. Alzouebi M, Goepel JR, Horsman JM, Hancock BW (2012) Primary thyroid lymphoma: the 40-year experience of a UK lymphoma treatment centre. Int J Oncol 40: 2075-2080. [Crossref]

12. Graff-Baker A, Roman SA, Thomas DC, Udelsman R, Sosa JA (2009) Prognosis of primary thyroid lymphoma: demographic, clinical, and pathologic predictors of survival in 1,408 cases. Surgery 146: 1105-1115. [Crossref]

13. Yoshino T, Omonishi K, Kobayashi K, Mannami T, Okada H, et al. (2000) Clinicopathological features of gastric mucosa associated lymphoid tissue (MALT) lymphomas: high grade transformation and comparison with diffuse large B cell lymphomas without MALT lymphoma features. J Clin Pathol; 53: 187-190. [Crossref]

14. Zucca E, Conconi A, Pedrinis E, Cortelazzo S, Motta T, et al. (2003) Nongastric marginal zone B-cell lymphoma of mucosa-associated lymphoid tissue. Blood 101: 2489-2495. [Crossref]

15. Thieblemont C, Bastion Y, Berger F, Rieux C, Salles G, et al. (1997) Mucosa-associated lymphoid tissue gastrointestinal and nongastrointestinal lymphoma behavior: analysis of 108 patients. J Clin Oncol 1997; 15: 1624-1630. [Crossref]

16. Sasai K, Yamabe H, Haga H, Tsutsui K, Dodo Y, et al. (1996) Non-Hodgkin's lymphoma of the thyroid. A clinical study of twenty-two cases. Acta Oncol 35: 457-462. [Crossref]

17. Verma D, Puri V, Agarwal S, Bhaskar A (2014) Primary thyroid lymphoma: A rare disease. J Cytol 31: 218-220. [Crossref]

18. Katna R, Shet T, Sengar M, Menon H, Laskar S, et al. (2013) Clinicopathologic study and outcome analysis of thyroid lymphomas: experience from a tertiary cancer center. Head Neck 35: 165-171. [Crossref]

19. Sakorafas GH, Kokkoris P, Farley DR (2010) Primary thyroid lymphoma (correction of lympoma): diagnostic and therapeutic dilemmas. Surg Oncol 19: e124-129. [Crossref]

20. Cha H, Kim JW, Suh CO, Kim JS, Cheong JW, et al. (2013) Patterns of care and treatment outcomes for primary thyroid lymphoma: a single institution study. Radiat Oncol J 31: 177-184. [Crossref]

21. Wang Z, Fu B, Xiao Y, Liao J, Xie P (2015) Primary thyroid lymphoma has different sonographic and color Doppler features compared to nodular goiter. J Ultrasound Med 34: 317-323. [Crossref]

22. Kim EH and Kim JY (2014) Aggressive primary thyroid lymphoma: imaging features of two elderly patients. Ultrasonography 33: 298-302. [Crossref]

23. Swart GJ, Wright C, Brundyn K, Mansvelt E, du Plessis M, et al. (2007) Fine needle aspiration biopsy and flow cytometry in the diagnosis of lymphoma. Transfus Apher Sci 37: 71-79. [Crossref]

24. Swart GJ, Wright CA (2010) The utilization of fine needle aspiration biopsy (FNAB) and flow cytometry (FC) in the diagnosis and classification of non-Hodgkin B-cell and T-cell lymphomas. Transfus Apher Sci 42: 199-207. [Crossref]

25. Ota H, Ito Y, Matsuzuka F, Kuma S, Fukata S, Morita S, et al. (2006) Usefulness of ultrasonography for diagnosis of malignant lymphoma of the thyroid. Thyroid 16: $983-$ 987. [Crossref]

26. Orita Y, Sato Y, Kimura N, Marunaka H, Tachibana T, et al. (2014) Characteristic ultrasound features of mucosa-associated lymphoid tissue lymphoma of the salivary and thyroid gland. Acta Otolaryngol 134: 93-99. [Crossref]

27. Nam M, Shin JH, Han BK, Ko EY, Ko ES, et al. (2012) Thyroid lymphoma: correlation of radiologic and pathologic features. J Ultrasound Med 31: 589-594. [Crossref]

28. Moon WJ, Baek JH, Jung SL, Kim DW, Kim EK, et al. (2011) Ultrasonography and the ultrasound-based management of thyroid nodules: consensus statement and recommendations. Korean J Radiol 12: 1-14. [Crossref]

29. Ramelli F, Studer H, Bruggisser D (1982) Pathogenesis of thyroid nodules in multinodular goiter. Am J Pathol 109: 215-223. [Crossref]

30. Gokdogan O, Koybasioglu A, Ismail E, Erol T, Alagoz G, et al. (2016) Hoarseness: an unusual presentation of primary thyroid lymphoma with laryngeal infiltration. Braz J Otorhinolaryngol 82: 737-740. [Crossref]

Copyright: (C2018 Zhang P. This is an open-access article distributed under the terms of the Creative Commons Attribution License, which permits unrestricted use, distribution, and reproduction in any medium, provided the original author and source are credited. 